\title{
Biología de la mosca minadora Liriomyza huidobrensis Blanchard (Diptera, Agromyzidae)*
}

\author{
Alfonso D. Lizárraga **
}

\section{RESUMEN}

Utilizando esquejes de papa del cv. Mariva, se obtuvieron ciclos de desarrollo de Liriomyza huidobrensis de 40 días en invierno, 25 días en primavera y 19 días en verano. La longevidad de las hembras fue mayor a la de los machos, presentando un período de pre-oviposición y una capacidad de reproducción de 252 huevos promedio durante la primavera. Las picaduras de alimentación aumentaron conforme aumentó la temperatura.

Palabras Claves Adicionales: Mosca minadora, biología, ciclos de desarrollo, papa.

\section{SUMMARY}

\section{Biology of Leaf Miner Fly Liriomyza huidobrensis Blanchard (Diptera, Agromyzidae)}

Using potatoes cutting of cv. Mariva the development cycles of Liriomyza huidobrensis of 40 days in the winter, 25 in the spring and 19 in the summer were obtained. Females longevity was greater than in males; there was noticed that a pre-oviposition period and the reproduction capability was of 252 eggs average in the spring. Feeding punctures increased as temperature increased.

Additional Index Words: Leaf miner fly, biology, development cycles, potato.

Aceptado para publicación: Febrero 5, 1991

* Trabajo financiado por el Programa Andino Cooperativo de Investigación en Papa (PRACIPA).

** Biólogo, Centro de Introducción y Cría de Insectos Útiles (CICIU). Apartado Postal 140008. Lima, Perú. 
La mosca minadora Liriomyza huidobrensis B. es una de las principales plagas en el cultivo de la papa en la Costa Central del Perú, así como en algunos otros países del continente americano.

Las larvas se alimentan del mesófilo de las hojas dejando intactas las capas externas o epidemiales. Las hojas afectadas presentan "minas", perdiendo de esta manera su capacidad fotosintética, defoliándose total o parcialmente (2).

Spencer y Steyskal en 1986 (11) mencionan a L. huidobrensis como uno de los agromyzidos que mayor daño causa a la agricultura; Raman en 1988 (9) menciona la presencia de L. huidobrensis en Chile, Perú, Argentina, Colombia y Estados Unidos.

Herrera en 1963 (3) mostró importantes observaciones biológicas estacionales realizadas en Cañete (Perú); otras observaciones similares se han realizado a temperaturas constantes: Aguilera (1) a $19.9 \pm 1.8^{\circ} \mathrm{C}$; Palacios y Cisneros (6) y Parrella y Bethke (7) a $26.7 \pm 0.5^{\circ} \mathrm{C}$; Prando y Da Cruz (8) de 25 a $26^{\circ} \mathrm{C}$.

Por lo expuesto, la poca información de observaciones en la biología de $L$. huidobrensis, motivaron a realizar el estudio de la biología del insecto en diferentes épocas y temperaturas, con la finalidad de analizar su crianza, así como la de sus principales controladores biológicos en el cultivo de papa.

\section{MATERIALES Y METODOS}

El estudio se llevó a cabo en el Centro de Introducción y Cría de Insectos Útiles (CICIU) del Instituto Nacional de Investigaciones Agropecuarias y Agroindustriales (INIAA), en Vitarte, Lima-Perú. Se realizaron observaciones de la biología de $L$. huidobrensis en tres épocas del año: invierno (14.6 $\pm 0.8^{\circ}$ $\mathrm{C}$ y $88.4 \pm 2.9 \% \mathrm{HR})$; primavera $\left(17.3 \pm 0.7^{\circ} \mathrm{C}\right.$ y $\left.88 \pm 3.3 \% \mathrm{HR}\right)$ y principios del verano $\left(20.4 \pm 0.9^{\circ} \mathrm{C}\right.$ y $\left.82.1 \pm 4 \% \mathrm{HR}\right)$.

Se colectaron hojas de papa infestadas por L. hudobrensis individualizando los puparios obtenidos, separando posteriormente 10 parejas de imagos emergidos en la misma fecha. Cada pareja se colocó en reposteros de plástico transparentes de un litro de capacidad, de 14 x 10 x 10 cm (5). 
Se utilizaron esquejes de papa del cv. Mariva, trasplantados en vasos de plástico descartables. Cada 24 horas se introdujo un nuevo esqueje de papa en cada jaula, cortándose previamente los foliolos. No se hizo esta operación en tres de ellos $(5 \mathrm{~cm} \mathrm{c} / \mathrm{u})$, con el objeto de facilitar el conteo diario de huevos y picaduras de alimentación. En los foliolos se determinó el ritmo de oviposición, el número de picaduras de alimentación, la capacidad de oviposición y la longevidad.

Se separaron 50 huevos para determinar la incubación, 15 larvas para determinar la duración de cada estadio larval (midiendo diariamente la longitud del gancho mandibular) y 15 puparios colectados sobre papel toalla colocado alrededor del esqueje.

La longevidad de los adultos se evaluó bajo tres regímenes alimenticios: el primero sin utilizar aumento ni sustrato alguno; el segundo utilizando solamente una solución compuesta por miel de abejas y agua y en el tercero se utilizó únicamente esquejes de papa. En todos los casos se asperjó agua cada 24 horas y se utilizaron 10 individuos por cada repetición en cada uno de los tratamientos.

Se colectaron 100 especímenes adultos del campo; 100 emergidos en el laboratorio, originados por huevos colocados por una sola hembra y 100 especímenes emergidos en el laboratorio, originados por huevos colocados por diversas hembras, con la finalidad de determinar la relación de sexos (proporción sexual).

\section{RESULTADOS Y DISCUSIÓN}

La hembra de L. huidobrensis se diferencia del macho por presentar un ovipositor prominente al final del abdomen, y ser de mayor tamaño. Los adultos son de color negro, con el escutelo, frons, halterios, genas y patas de color amarillo.

La hembra, utilizando el ovipositor, realiza perforaciones en la hoja moviéndolo de atrás hacia adelante e inoculando solamente un huevo por cada oviposición. La hembra oviposita en el envés de las hojas, probablemente para proteger los huevos de la influencia de los rayos solares.

Generalmente en el haz de las hojas, con ayuda del ovipositor, las hembras realizan perforaciones conocidas como "picaduras de alimentación". Luego de realizarlas, la hembra retrocede, extiende la proboscis absorbiendo el jugo de la planta que queda expuesto posterior a la picadura. De forma 
similar, tanto Aguilera (1) como Prando y Da Cruz (8) exponen resultados referidos a la oviposición y a la forma de alimentación.

El huevo ovalado, algo alargado, sin ornamentos y de color opalescente a lechoso, mide 0.29 x $0.16 \mathrm{~mm}$. La larva es pequeña llegando aproximadamente a tres milímetros de longitud. Sin embargo, su tamaño varía de acuerdo a la cantidad y calidad del alimento ingerido. Se toma en cuenta la dimensión de los ganchos mandibulares como un patrón para la determinación de los tres estadios larvales.

Los ganchos mandibulares tienen un promedio de $0.125 \mathrm{~mm}$ en el primer estadio larval, $0.216 \mathrm{~mm}$ en el segundo y $0.310 \mathrm{~mm}$ en el tercero. El último estadio cuenta con dos fases, una de alimentación dentro de la hoja y otra de post-alimentación (período prepupal), realizado generalmente en el exterior de la hoja.

La larva es blanquecina, más o menos cilíndrica, ápoda y sin ojos; construye "minas" serpenteantes en el interior de la hoja consumiendo el clorenquima de ésta. Es bastante activa y prefiere alimentarse a lo largo de las nervaduras del foliolo. En este caso, hay que tener presente que una misma especie de mosca minadora puede realizar diversas formas de mina, de acuerdo a la planta que infesta.

La pupa de L. huidobrensis se encuentra protegida por un pupario de color café claro a oscuro, más o menos cilindrico y mide 2.1 x 0.9 mm.

\section{Duración del ciclo biológico}

La duración del ciclo biológico de Liriomyza huidobrensis disminuye a medida que se eleva la temperatura (Tabla 1).

En el invierno, el huevo incuba en seis días, la larva desarrolla en 13, la pupa en 21, completando el ciclo biológico en 40 días. Durante la primavera, el ciclo biológico disminuye sustancialmente, incubando el huevo en 2.9 días, la larva desarrolla en 11 aproximadamente, igual que la pupa completando el ciclo biológico en 25 días. A principios del verano se aprecia un ciclo biológico de menor duración, equivalente a 19 días, con una incubación del huevo de 3 días, desarrollo larval en 9 días y la pupa en 7 días.

El período de incubación coincide con los reportados por diversos autores, sin embargo, Sánchez (10) indica un rango mayor con una duración de 2 a 12 días. 
Tabla 1. Duración en días de los estados de desarrollo de Liriomyza huidobrensis, en tres épocas del año en Vitarte, Lima-Perú, llevadas a cabo en laboratorio.

\begin{tabular}{llll}
\hline Estado & Invierno & Primavera & Verano \\
\hline Huevo & $6 \pm 0$ & $3 \pm 0.5$ & $3 \pm 0.5$ \\
Larva I & $3 \pm 0.7$ & $4 \pm 0.4$ & $3 \pm 0.4$ \\
Larva II & $5 \pm 0.6$ & $3 \pm 0.4$ & $3 \pm 0.4$ \\
Larva III & $5 \pm 1.0$ & $4 \pm 0.4$ & $4 \pm 04$ \\
Pupa & $21 \pm 1.0$ & $12 \pm 3.0$ & $7 \pm 1.0$ \\
\hline Total: & & & \\
Huevo a adulto (días) & $\mathbf{4 0}$ & $\mathbf{2 5}$ & $\mathbf{1 9}$ \\
\hline
\end{tabular}

Otros autores $(7,8)$ han reportado la duración del estado larval en menos de 7.4 días a $25^{\circ} \mathrm{C}$, confirmando el hecho de que el aumento de temperatura es el factor de la disminución del tiempo del ciclo biológico.

\section{Longevidad}

La longevidad de los adultos en los diversos tratamientos se muestra en la Tabla 2. Los individuos que no fueron alimentados, vivieron sólo algunos días como era de esperar; las moscas alimentadas tuvieron, en cambio, una longevidad notoriamente mayor.

Tabla 2. $\quad$ Longevidad en días de adultos de Liriomyza huidobrensis estimada en tres épocas del año usando tres tipos regímenes de alimentación en condiciones de laboratorio de Vitarte, Lima-Perú.

\begin{tabular}{lcccccc}
\hline Alimento & \multicolumn{2}{c}{ Invierno } & \multicolumn{2}{c}{ Primavera } & \multicolumn{2}{c}{ Verano } \\
& H* $^{*}$ & $\mathbf{M}^{*}$ & H & M & H & M \\
\hline Sin alimento $\left({ }^{\circ}\right)$ & 5 & 2 & 4 & 3 & 4 & 3 \\
Miel / Agua $\left({ }^{\circ}\right)$ & 33 & 14 & 31 & 15 & 19 & 13 \\
Hospedero/ Agua $\left({ }^{\circ}{ }^{\circ}\right)$ & 18 & 4 & 24 & 7 & 19 & 5 \\
\hline
\end{tabular}

$\begin{array}{ll}\circ \text { Virgen } & * \text { H: Hembra; M : Macho } \\ \circ \text { O Apareado } & \end{array}$


Cuando las moscas no eran alimentadas, tanto las hembras como los machos, tuvieron una longevidad que no sobrepasaba los 5 días, repitiéndose estos resultados en todas las épocas en las que se realizaron las pruebas.

El alimento que determinó una mayor longevidad de las moscas fue el compuesto por miel de abejas y agua; las hembras tuvieron, en este caso, una longevidad entre 18 y 32 días, siendo menor durante el verano; los machos entre 13 y 15 días.

Cuando las moscas se criaron en los esquejes de papa, las hembras tuvieron una longevidad entre 18 y 23 días, mientras que los machos entre 4 y 7 días.

La dieta de miel de abejas y agua incrementó la longevidad notablemente en comparación a la dieta compuesta solamente por el alimento natural de hojas de papa. La concentración de proteínas y carbohidratos de la miel, aparentemente resultan ser nutritivamente de mayor calidad y/o cantidad a los extractos obtenidos de la hoja de papa.

\section{Período de pre-oviposición y oviposición}

El período de pre-oviposición es fundamental que se cumpla (Tabla 3). Durante el invierno, la pre-oviposición fue cercana a los 4 días y la oviposición a los 11 días; en la primavera, la pre-oviposición fue de 2 días y la oviposición de 19 días. A principios del verano la pre-oviposición fue de 4.5 días y la oviposición de 10.4 días.

Durante la primavera se aprecia una tendencia a disminuir la pre-oviposición, sin embargo, en el período de oviposición se observa un incremento (Figura $1)$.

Los períodos de pre-oviposición y oviposición son más o meno., similares antes y después del clima primaveral o sea durante el invierno y principios del verano. Esto es una indicación del incremento de la población de la mosca minadora durante la primavera en el campo (Figura 1).

\section{Capacidad de reproducción y alimentación}

La capacidad de una hembra para producir huevos durante los períodos de oviposición, así como la capacidad de las hembras de realizar picaduras de alimentación se muestra en la Tabla 3. 


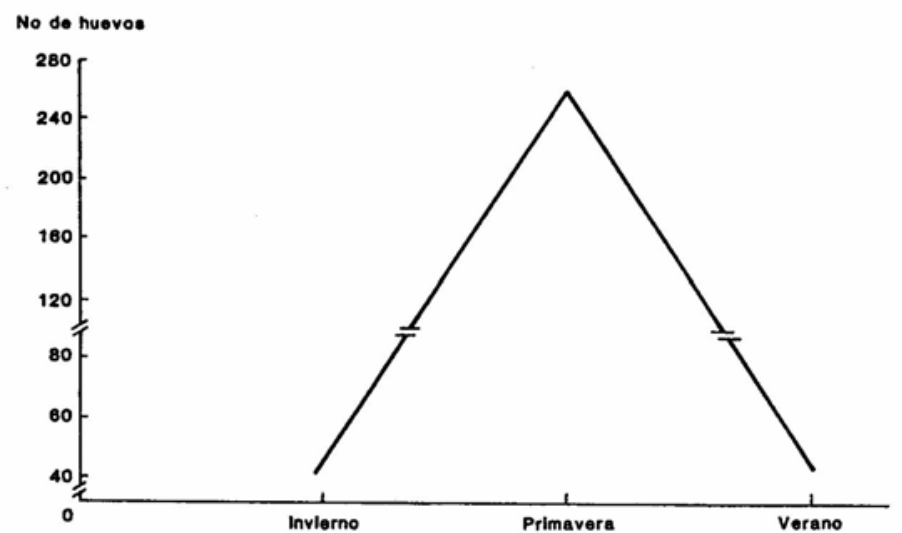

Figura 1. Capacidad de oviposición de L. huidobrensis.

Tabla 3. Duración, en días, en tres épocas del año, de los períodos de pre-oviposición y oviposición, capacidad de oviposición y de realizar picaduras de alimentación de Liriomyza huidobrensis, determinadas bajo condiciones del laboratorio, Vitarte, Lima, Perú.

\begin{tabular}{lccc}
\hline & Invierno & Primavera & Verano \\
\cline { 2 - 4 } Pre-oviposición & $4 \pm 1$ & $2 \pm 6$ & $5 \pm 2$ \\
Oviposición & $11 \pm 6$ & $19 \pm 7$ & $10 \pm 5$ \\
No. de huevos & $45 \pm 35$ & $252 \pm 115$ & $42 \pm 21$ \\
$\begin{array}{l}\text { No. de picaduras } \\
\text { de alineación }\end{array}$ & $1875 \pm 775$ & $3055 \pm 1237$ & $3473 \pm 894$ \\
\hline
\end{tabular}

Resalta la capacidad reproductiva durante la primavera de $252 \pm 115$ huevos por hembra. Durante las estaciones de invierno y verano la capacidad de reproducción disminuye, obteniéndose no más de 45 huevos promedio.

En trabajos realizados sobre papa el promedio reportado para la capacidad de reproducción es bastante menor. Jara y Alvites (4) mencionan un rango de 62 a 65 huevos; Palacios y Cisneros (6) hacen referencia a 62.7 huevos en promedio. Sin embargo, Prando y Da Cruz (8) utilizando frijol como sustrato, reportan 113 huevos promedio. 
La cantidad de picaduras de alimentación se incrementó a medida que aumentó la temperatura. Durante el invierno, las hembras realizaron 1875 picaduras, 3055 en la primavera y 3473 durante los inicios del verano. Prando y Da Cruz (8) reportan 3168 picaduras de alimentación a $26^{\circ} \mathrm{C}$, resultados muy similares a los obtenidos en este trabajo durante la primaveraverano.

Es necesario, sin embargo, tomar en cuenta la preferencia del insecto en su alimentación ya que se ha observado la existencia de preferencia por determinada planta, al momento de realizar las picaduras (7).

\section{Ritmo de oviposición}

Durante la primavera existe una mayor oviposición, preferentemente durante el cuarto y decimoquinto día de longevidad de las hembras, observando una oviposición promedio de 25 huevos diarios (Figura 2). Este resultado es similar al obtenido por Aguilera (1) quien registró un máximo de 25 posturas por hoja de alfalfa a $20^{\circ} \mathrm{C}$ y $85 \% \mathrm{HR}$.

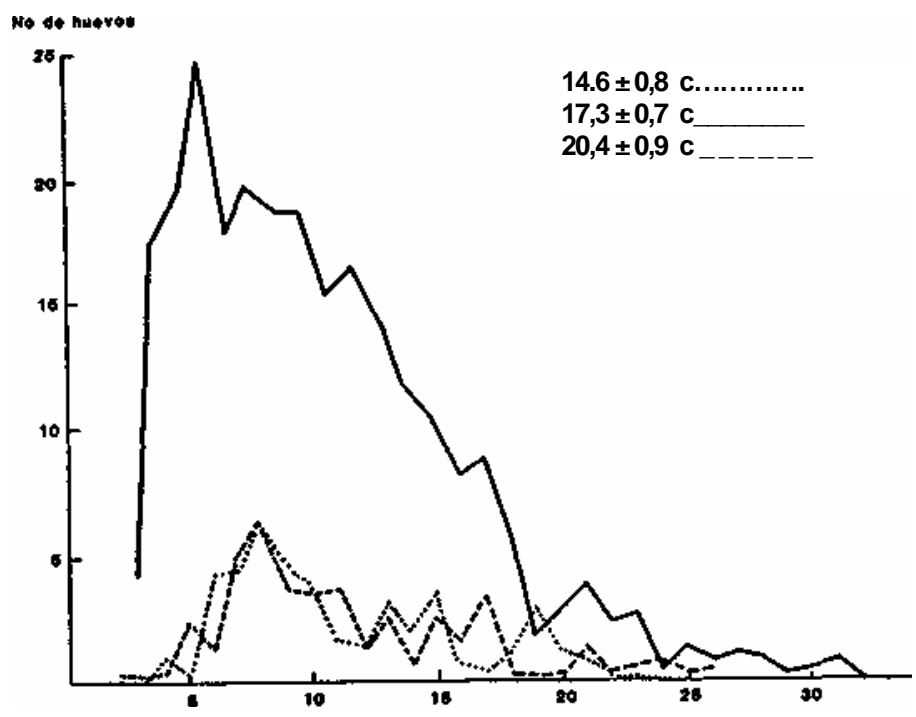

Dias

Figura 2. Ritmo de oviposición realizado por hembras de L. huidobrensis. 
Los datos obtenidos en invierno y a principios del verano son más o menos similares, sin llegar a alcanzar los resultados del período de primavera. Este resultado es un indicador del aumento de la población de mosca minadora durante la primavera y la notoria disminución posterior a esta estación, complementada por el aumento del parasitismo.

\section{Proporción Sexual}

Las hembras de $L$. huidobrensis se encuentran ligeramente en mayor proporción que los machos (Tabla 4) al igual que lo que se reporta en la literatura $(6,8)$.

Como el ciclo biológico de la mosca minadora disminuye a medida que aumenta la temperatura, sería lógico esperar el aumento de la población durante el verano; en la realidad sin embargo, sucede todo lo contrario.

Entre las respuestas biológicas que explican su variación poblacional (Figura 3) para la mayor capacidad de oviposición durante la primavera, y una muy baja oviposición durante el invierno y el verano están: (a) la pre-oviposición más larga en el verano y más corta durante la primavera y (b) la mayor duración de la longevidad y la oviposición en la primavera. Además durante el verano casi no se siembran los cultivos preferidos por la mosca minadora, como papa y frijol. Los pocos huevos ovipositados en el terreno encontrarán condiciones adversas para su desarrollo, explicarían el por qué de la mosca

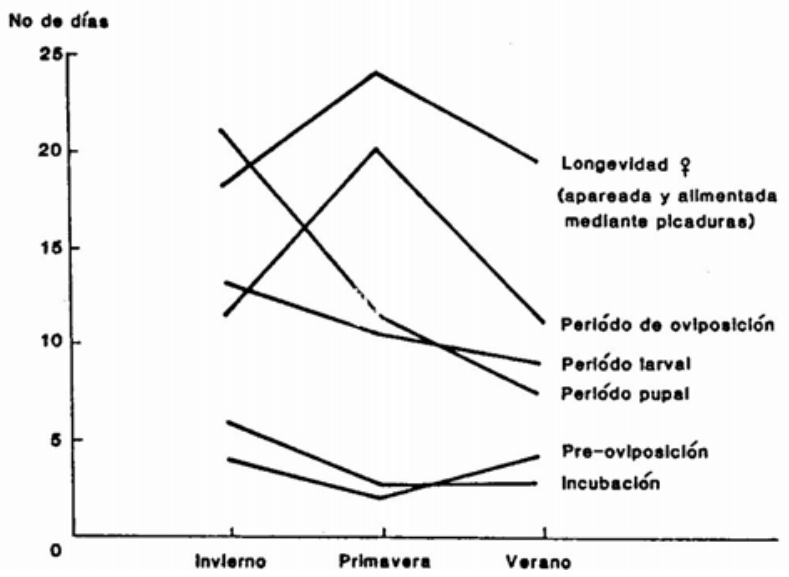

Figura 3. Períodos de desarrollo, oviposición y longevidad de L. huidobrensis. 
minadora Liriomyza huidobrensis desciende a niveles que no revisten mayor importancia durante el verano.

Tabla 4. Proporción sexual (hembra/macho) de Liriomyza huidobrensis estimadas con material obtenido de tres orígenes y conducido bajo condiciones del laboratorio, Vitarte, La Molina-Lima, Perú.

\begin{tabular}{lccc}
\hline & Invierno & Primavera & Verano \\
\cline { 2 - 4 } De campo & $1.4: 1.0$ & $1.3: 1.0$ & $1.0: 1.0$ \\
$\begin{array}{l}\text { De laboratorio al } \\
\text { azar }\end{array}$ & $1.3: 1.0$ & $1.2: 1.0$ & $1.9: 1.0$ \\
$\begin{array}{l}\text { De laboratorio } \\
\text { (una hembra) }\end{array}$ & $1.0: 1.0$ & $1.6: 1.0$ & $1.0: 1.0$ \\
\hline
\end{tabular}

\section{AGRADECIMIENTOS}

Al Ing. Cesar Vittorelli por la revisión del manuscrito y a PRACIPA por el apoyo brindado para la ejecución del presente trabajo. Al personal del CICIU por sus sugerencias y orientaciones.

\section{REFERENCIAS BIBLIOGRÁFICAS}

1. Aguilera, A.P. 1972. Biología de Liriomyza huidobrensis Frick (Dipt., Agromyzidae) y evaluación de los parásitos que emergen del puparium. IDESIA. 2:71-85.

2. Cisneros, F. 1980. Principios del Control de Plagas Agrícolas, 1a. Ed. LimaPerú. 120 pp.

3. Herrera, J. 1963. Problemas insectiles del cultivo de papa en el valle de Cañete. Rev. Per. Ent. 6(1): 1-9.

4. Jara, B.; Alvites, S. 1980. La mosca minadora de la papa Liriomyza huidobrensis (Blanchard), Dipt., Agromyzidae. Boletín Técnico $\mathrm{N}^{\circ}$ 6, Estación Experimental San Camilo. 10 pp.

5. Lizárraga, A.T. 1988. Biología de la mosca minadora Liriomyza huidobrensis B. (Dipt., Agromizydae). Informe Final (CICIU-PRACIPA). 15 pp.

6. Palacios. M.; Cisneros, F. 1980. Biología y comportamiento de la mosca minadora Liriomyza huidobrensis B. Resúmenes. XXIII Conv. Nac. Ent. Huacho. 62 pp. 
7. Parrella, M.P.; Bethke, JA. 1984. Biological studies of Liriomyza huidobrensis (Dip. Agromyzidae) on chrysanthemum, aster and pea. J. Econ. Entomol. 77(2):342-345.

8. $\quad$ Prando, H.F.; Da Cruz, F.Z. 1986. Aspectos a biología de Liriomyza huidobrensis B. (Dip. Agromyzidae) en laboratorio. An. Soc. Entomol. Brasil. 15(1): 77-88.

9. Raman, K.V. 1988. Manejo integrado de plagas de la papa en los países del tercer mundo. Circular CIP. 16(1): 1-9.

10. Sánchez, G. 1986. Principales plagas y enfermedades de la papa en el Perú, INIPA, Manual Técnico Nº 1.230 pp.

11. Spencer, KA.; Setyskal, G.C. 1986. Manual of the Agromyzidae (Diptera) of the United States. Agricultural Research Service, U.S. Department of Agriculture. 467 pp. 